\title{
six3 acts upstream of foxQ2 in labrum and neural development in the spider Parasteatoda tepidariorum
}

\author{
Magdalena Ines Schacht ${ }^{1,2} \cdot$ Christoph Schomburg ${ }^{1,3} \cdot$ Gregor Bucher $^{1}$ (B)
}

Received: 20 October 2019 / Accepted: 29 January 2020 /Published online: 10 February 2020

(C) The Author(s) 2020

\begin{abstract}
Anterior patterning in animals is based on a gene regulatory network, which comprises highly conserved transcription factors like six 3, pax6 and otx. More recently, foxQ2 was found to be an ancestral component of this network but its regulatory interactions showed evolutionary differences. In most animals, fox $Q 2$ is a downstream target of six 3 and knockdown leads to mild or no epidermal phenotypes. In contrast, in the red flour beetle Tribolium castaneum, foxQ2 gained a more prominent role in patterning leading to strong epidermal and brain phenotypes and being required for six 3 expression. However, it has remained unclear which of these novel aspects were insect or arthropod specific. Here, we study expression and RNAi phenotype of the single foxQ2 ortholog of the spider Parasteatoda tepidariorum. We find early anterior expression similar to the one of insects. Further, we show an epidermal phenotype in the labrum similar to the insect phenotype. However, our data indicate that foxQ2 is positioned downstream of six 3 like in other animals but unlike insects. Hence, the epidermal and neural pattering function of foxQ2 is ancestral for arthropods while the upstream role of fox $Q 2$ may have evolved in the lineage leading to the insects.
\end{abstract}

Keywords Spiders $\cdot$ Labrum $\cdot$ RNAi $\cdot$ Gene interaction

\section{Introduction}

Anterior patterning in animals is based on an anterior gene regulatory network (aGRN), which is built by a conserved

Magdalena Ines Schacht and Christoph Schomburg contributed equally to this work.

This article is part of the Special Issue "Crossroads in Spider Research evolutionary, ecological and economic significance."

Communicated by Matthias Pechmann

Electronic supplementary material The online version of this article (https://doi.org/10.1007/s00427-020-00654-9) contains supplementary material, which is available to authorized users.

Gregor Bucher

gbucher1@uni-goettingen.de

1 Department of Evolutionary Developmental Genetics, GZMB, University of Göttingen, Justus-von-Liebig-Weg 11, 37077 Göttingen, Germany

2 School of Biological and Chemical Sciences, Queen Mary University of London, Mile End Road, London E1 4NS, UK

3 Institut für Allgemeine Zoologie und Entwicklungsbiologie, Justus-Liebig-Universität Gießen, Heinrich-Buff-Ring 38, 35392 Giessen, Germany set of transcription factors and signalling pathways (Lowe et al. 2003; Marlow et al. 2014; Posnien et al. 2011; Sinigaglia et al. 2013; Steinmetz et al. 2010; Yaguchi et al. 2008; Tosches and Arendt 2013). However, functional studies revealed that the regulatory interactions within this ancestral patterning system have diverged between animals (Range and Wei 2016; Sinigaglia et al. 2013; Yaguchi et al. 2008; Kitzmann et al. 2017). One example is the function of the forkhead box transcription factor foxQ2. The forkhead box (Fox) family is a large family of transcription factors that holds many important regulatory roles in numerous developmental and homeostatic processes (Fritzenwanker et al. 2014; Uhlenhaut and Treier 2011; Benayoun et al. 2011; Carlsson and Mahlapuu 2002), and is widely represented in the animal kingdom (Kaestner et al. 2000). fox $Q 2$ is a member of this transcription factor family, which is present in all major lineages of the metazoans (Chevalier et al. 2006; Sinigaglia et al. 2013; Marlow et al. 2014; Tu et al. 2006; Yu et al. 2003; Lee and Frasch 2004). In deuterostomes, such as the cephalochordate Branchiostoma floridae (B. floridae) and the echinoderm Strongylocentrotus purpuratus (S. purpuratus), foxQ2 is expressed at the anterior pole from the late blastula stage (B. floridae) or mesomeric cleavage stage (S. purpuratus) onwards. In both cases, transcripts are 
still detected in larval stages in the anterior tip of the ectoderm (Yu et al. 2003; Tu et al. 2006; Yaguchi et al. 2008; Yankura et al. 2010; Range and Wei 2016). The three paralogs of foxQ2 in the hemichordate Saccoglossus kowalevski (S. kowalevskii) also share a largely apical expression pattern, while exhibiting temporal differences (Fritzenwanker et al. 2014). A conserved apical expression of fox $Q 2$ is also present in lophotrochozoans, such as the annelid Platynereis dumerilii (P. dumerilii) and the brachiopod Terebratalia transversa (T. transversa) (Santagata et al. 2012; Marlow et al. 2014), as well as in the early development of the insects Drosophila melanogaster (D. melanogaster) and Tribolium castaneum (T. castaneum) (Lee and Frasch 2004; Kitzmann et al. 2017). Additionally, in non-bilaterian species, such as the cnidarians Nematostella vectensis ( $N$. vectensis) and Clytia hemispherica (C. hemispherica), fox $Q 2$ expression is restricted to the aboral pole of the embryos (Chevalier et al. 2006; Sinigaglia et al. 2013), supporting the hypothesis of homology between the cnidarian aboral pole and the bilaterian anterior pole (Sinigaglia et al. 2013). Interestingly, while orthologs are found in fish, the foxQ2 gene was apparently lost from the genomes of amphibians and mammalians.

foxQ2 is expressed in neurogenic regions of different species (Kitzmann et al. 2017; Hunnekuhl and Akam 2014; Marlow et al. 2014; Santagata et al. 2012). In $P$. dumerili, fox $Q 2$ and six3 are expressed in the apical plate including sensory-neurosecretory cells (Marlow et al. 2014). Similarly, in the myriapod Strigamia maritima ( $S$. maritima), nested domains of fox $Q 2$ and six 3 expression were identified in neural precursors of the embryonic head (Hunnekuhl and Akam 2014). Both these transcription factors pattern anterior neurogenic domains during the embryonic development of the brachiopod T. transversa (Santagata et al. 2012). In the red flour beetle, Tc-foxQ2positive cells contribute to the central complex and the gene is required for central brain development (He et al. 2019). In $T$. castaneum, an extended GRN for anterior head development has been proposed in which Tc-six3 and Tc-foxQ2 hold upstream positions (Kitzmann et al. 2017). They mutually regulate each other forming an upstream core unit, which regulates several downstream targets. Those targets are required for the proper formation of anterior structures, like the head placode, neurogenic ectoderm, labrum and stomodeum, and are therefore important for the development of the head and brain (Kitzmann et al. 2017). Intriguingly, no or only mild epidermal effects were found in foxQ2 knockdown embryo in other animals, while in T. castaneum, a strong effect on epidermal patterning was observed (Kitzmann et al. 2017). This finding correlated with the downstream role of fox $Q 2$ in most analysed species except for T. castaneum. However, it has remained unclear which of these aspects are ancestral for arthropods and which may be insect specific.
In order to gain insights from a basal euarthropod, we studied the foxQ2 expression, its RNAi phenotype and its interaction with six 3 in the spider $P$. tepidariorum. We find that the epidermal labrum phenotype is conserved but that spider $P t$ fox 22 appears not to regulate either of the two Pt-six 3 paralogs.

\section{Material and methods}

\section{Phylogenetic analysis}

The Pt-foxQ2 sequence (aug3.g4586.t1) was identified by performing a BLAST search using the translated nucleotide query of FoxQ2 from D. melanogaster (declared on FlyBase as fd102C, FlyBase ID: FBgn0039937) in the P. tepidariorum genomic gene predictions (Schwager et al. 2017). The 20 bestscoring sequences were combined with published protein sequences of FoxQ2, as well as the protein sequence of FoxP of D. melanogaster and Danio rerio, which were retrieved from Fritzenwanker et al. (2014). All protein sequences were subsequently used to perform a protein alignment with default settings using Clustal Omega (Sievers et al. 2011; Sievers and Higgins 2014). Phylogeny was inferred based on this alignment using MrBayes 3.2.0 (Ronquist et al. 2012), which was sampled from a total of 1836 runs using the Wag model of amino acid substitution.

\section{Animal culture and gene cloning}

Embryos and adults of $P$. tepidariorum were obtained from the stock in Göttingen. Spider were kept and fed as previously described (Turetzek et al. 2015; Pechmann et al. 2017). Embryos used for in situ hybridization (ISH) experiments were staged, collected and fixed as previously described (Hilbrant et al. 2012; Mittmann and Wolff 2012; Posnien et al. 2014). The P. tepidariorum genes six3.1 (NCBI GenBank: AB605265.1) and six3.2 (NCBI GenBank: AB605266.1) used in this work were already published in Schomburg et al. (2015). The identified Pt-foxQ2 sequence (NCBI GenBank: MN567069) was amplified from cDNA (fwd primer: ${ }^{5}$ 'CGCTACTGACCAGAACCCTTT ${ }^{3}$, rev primer: ' ${ }^{\text {'TAAGGAAGCAGCGGATGACCA }}{ }^{3}$ ' amplified a 979 bp long sequence) and cloned into the pJet1.2 vector (Thermo Fisher Scientific).

\section{Probe synthesis and in situ hybridization}

Pt-foxQ2 antisense mRNA probes for ISHs were synthesized from the entire cloned sequence (979 bp) with the DIG RNA labelling Mix using the T7 RNA polymerase (both Roche, DIG RNA labelling Mix with Cat.-No.: 11277073910 and T7 Polymerase with Cat.-No.: 10881767001). The Pt-six3.1 
and Pt-six3.2 antisense mRNA probes were those used in Schomburg et al. (2015) and are of 1439 bp and 1328 bp length, respectively. ISHs and nuclear SYTOX® Green staining in P. tepidariorum embryos were carried out as described previously (Prpic et al. 2008; Pechmann and Prpic 2009).

\section{dsRNA synthesis and RNA interference}

The complete cloned sequence for Pt-foxQ2, Pt-six3.1 and Ptsix3.2 (see details above) was amplified using vector specific primers combined with the T7 promoter sequence ${ }^{5}$ 'GAAT TGTAATACGACTCACTATAGG ${ }^{3}$ ) in standard PCR and served as a template for the in vitro transcription. Double stranded RNA (dsRNA) of each gene was synthesized using the Ambion ${ }^{\circledR}$ T7 MEGAscript ${ }^{\circledR}$ Kit (Life Technologies, Carlsbad, CA, USA). Precipitation of the transcribed dsRNAs and the injection procedure was performed as described previously (Akiyama-Oda and Oda 2006; Turetzek et al. 2015). For Pt-foxQ2, Pt-six3.1 and Pt-six3.2, $2.5 \mu \mathrm{l}$ of dsRNA $(4 \mu \mathrm{g} / \mu \mathrm{l})$ was injected into sexually mature adult female spiders using borosilicate injection needles. Females were treated with 5 injections in total, whereby females were injected every second day and mated after the third injection. The same injection regime was applied for the negative control spiders that were injected with injection buffer only. Previous studies used GFP as a control injections which result in very few unspecific effects in RNAi embryos (Schwager et al. 2009; Paese et al. 2018). Therefore, we decided to use injection buffer only. Each female was kept and fed in the same way. The first four cocoons of each spider were collected, opened and kept in test tubes provided with a damp tissue to maintain humidity, essential for proper development. Half of each cocoon was fixed, whereas the other half could develop. During development, the embryos were checked regularly under the stereomicroscope and individual embryos of each cocoon were analysed in Voltalef® oil. All embryos were screened for any visible phenotypes. For the analysis of the embryos, batch 2-4 were merged because they had similar strong effects while batch 1 showed less penetrance.

\section{Microscopy and imaging}

Images of $P$. tepidariorum embryos were taken with a Leica M205 FA binocular (Leica Microsystems, Wetzlar, Germany) and recorded with the ImagePro v.7.0 software. Images were taken with fluorescent and white light simultaneously. After white correction, the green SYTOX ${ }^{\circledR}$ appears light blue, while the DIG staining appears dark blue, thus allowing staining and morphology to be shown in the same picture. Images were corrected for colour values and brightness with the Adobe Photoshop® CS6 software (version 9.0).

\section{Results}

\section{P. tepidariorum possesses one homolog of foxQ2}

Large-scale duplication of genes in spiders has been reported in P. tepidariorum before (Pechmann et al. 2015; Turetzek et al. 2015; Schomburg et al. 2015; Schwager et al. 2017; Leite et al. 2018). Therefore, we wanted to identify the total number of fox $Q 2$ orthologs in P. tepidariorum. We performed a multiple sequence alignment with FoxQ2 protein sequences of different metazoan species and generated a phylogenetic tree based on Bayesian analysis. The phylogenetic tree (Fig. 1) shows that the spider's translated transcript aug3.g4586.t1 clusters with all FoxQ2 sequences from the different species and is therefore indeed the spider fox $Q 2$ ortholog. None of the other identified $P$. tepidariorum sequences cluster with $P t$-FoxQ2 or any other analysed metazoan FoxQ2 sequence, indicating that there is only one homolog of foxQ2 in the spider (shown in red, Fig. 1).

\section{Pt-foxQ2 shows conserved expression in the anterior region of the embryo}

Previous studies in different metazoan species showed that fox $Q 2$ is expressed in the anterior regions during embryonic development (Yu et al. 2003; Lee and Frasch 2004; Tu et al. 2006; Marlow et al. 2014; Fritzenwanker et al. 2014; Kitzmann et al. 2017) but expression analysis of foxQ2 in chelicerates had been missing. Here, we analysed the expression of Pt-foxQ2 during spider embryogenesis (see Mittmann and Wolff, 2012 for detailed description of morphology during embryogenesis and Schomburg et al. 2015 for description of anterior structures for embryonic stages 10 to 13). We found that expression of Pt-foxQ2 started at stage 7 , when the germ band became evident, spanning the anterior rim of the germ band as a broad stripe (arrowhead in Fig. 2a; see Fig. S1 for stages 3-6, devoid of Pt-foxQ2 staining). At stage 8,Pt-fox $Q 2$ was bilaterally expressed in the anterior part of the pre-cheliceral region in one larger domain (white arrowheads in Fig. 2b) and an emerging smaller domain posterior-laterally to it (black arrowheads in Fig. 2b). Expression of $P t$-foxQ2 showed a tripartite domain on each side of the pre-cheliceral region at stage 9 with an additional domain on both sides of the anterior non-neurogenic ectoderm (asterisks in Fig. 2c; the non-neurogenic ectoderm in the precheliceral lobes is all ectoderm along the rim of the head lobes outside of the anterior and lateral furrows. This area is free of neural precursor invagination sites - see Fig. S2 for a scheme of a stage 11 embryo and Schomburg et al. 2015 for extensive description from stage 10 to 14). At stage 10, these three domains were localized in a different pattern. Transcripts of Pt-foxQ2 in the non-neurogenic ectoderm became localized more anteriorly (asterisks in Fig. 2d-f), while the median 


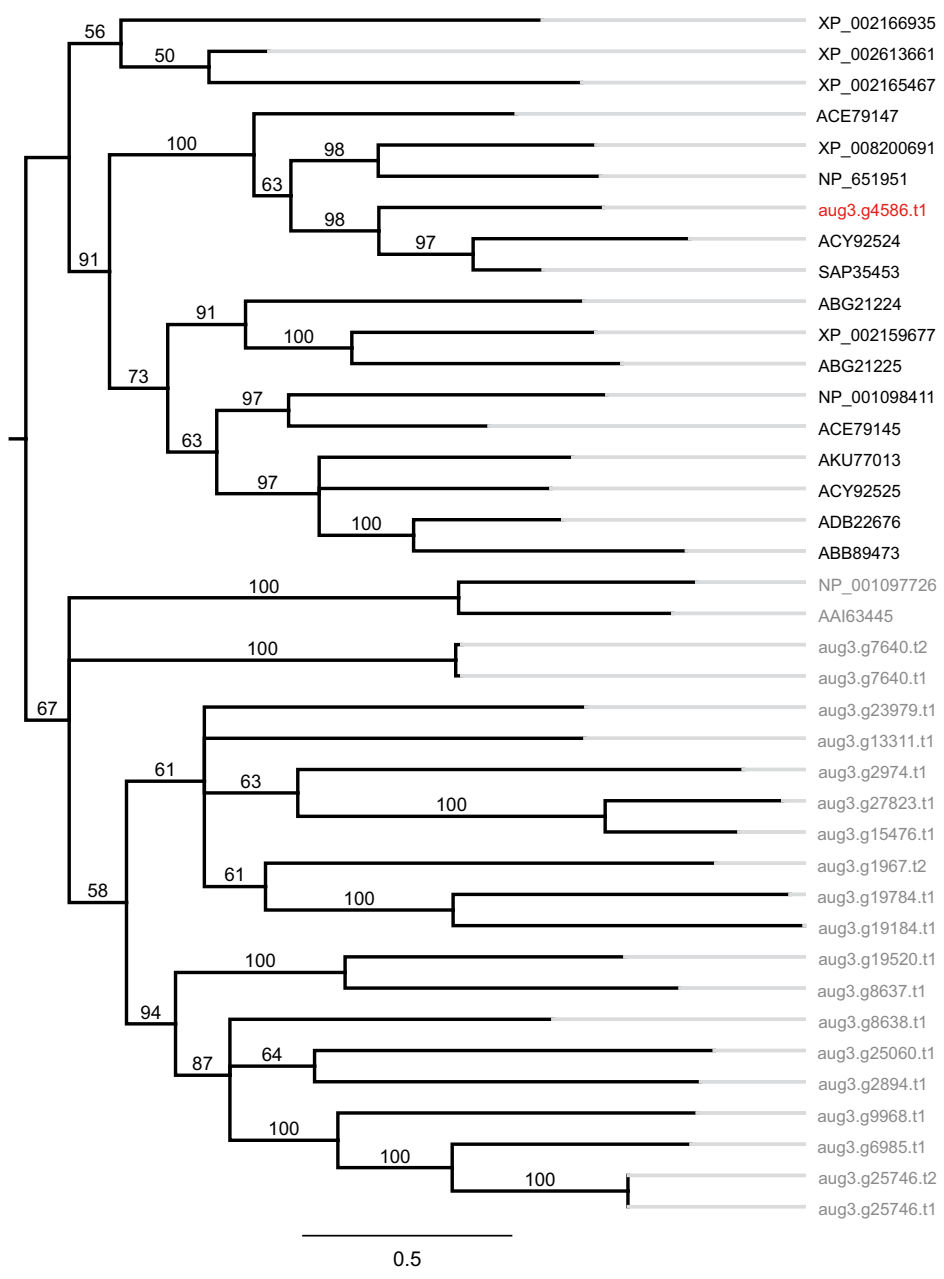

PREDICTED: Forkhead box protein C1-like - Hydra vulgaris Hypothetical protein BRAFLDRAFT 251612, partial - Branchiostoma floridae PREDICTED: Similar to predicted protein - Hydra magnipapillata Winged helix forkhead transcription factor FoxQ2c - Branchiostoma floridae PREDICTED: Protein lin-31 - Tribolium castaneum Forkhead domain 102C - Drosophila melanogaster Parasteatoda tepidariorum Forkhead box Q/D-like protein transcription factor - Saccoglossus kowalevskii FoxQ2, partial - Glomeris marginata

Forkhead box-containing transcription factor FoxQ2a - Clytia hemisphaerica PREDICTED: Forkhead box protein D2-like - Hydra vulgaris

Forkhead box-containing transcription factor FoxQ2b - Clytia hemisphaerica Forkhead box Q2 - Danio rerio

Winged helix forkhead transcription factor FoxQ2a - Branchiostoma floridae FoxQ2 - Priapulus caudatus

Forkhead box Q2-like transcription factor - Saccoglossus kowalevskii Fork head box Q2/QM transcription factor - Saccoglossus kowalevskii Forkhead transcription factor Q2 - Strongylocentrotus purpuratus Forkhead box P - Drosophila melanogaster

Forkhead box P1b - Danio rerio

Parasteatoda tepidariorum

Parasteatoda tepidariorum

Parasteatoda tepidariorum Parasteatoda tepidariorum

Parasteatoda tepidariorum Parasteatoda tepidariorum

Parasteatoda tepidariorum Parasteatoda tepidariorum

Parasteatoda tepidariorum Parasteatoda tepidariorum Parasteatoda tepidariorum Parasteatoda tepidariorum Parasteatoda tepidariorum Parasteatoda tepidariorum Parasteatoda tepidariorum

Parasteatoda tepidariorum Parasteatoda tepidariorum

Parasteatoda tepidariorum Parasteatoda tepidariorum
Fig. 1 Phylogenetic tree showing the P. tepidariorum FoxQ2 homolog. The $P$. tepidariorum predicted protein aug3.g4586.t1 (shown in red) clusters with the other FoxQ2 sequences and no other paralog was

domains split into three distinct domains (white arrowheads in Fig. 2d). The lateral expression in the neurogenic ectoderm splits into two domains between the edge of the head lobes and the prospective labrum (black arrowheads in Fig. 2d). At stage 11, the most lateral expression domains remained at the end of the anterior furrow (black arrowheads in Fig. 2e), while the median expression domain shifted posteriorly along with the stomodeum and labrum. The former domains fused into one (white arrowheads in Fig. 2e). These domains split up again at stage 12 with one part remaining in the region of the labrum (black arrowhead in Fig. 2f), while the other shifted further towards the posterior at stage 12 (white arrowheads in Fig. 2f). During stage 13, the non-neurogenic ectoderm started to grow over the brain region (Fig. $2 \mathrm{~g}$ ) and thereby covered the expression domains of Pt-foxQ2. At stage 14, Pt-foxQ2 expression was found in a complex spotted pattern in the brain region and at the base of the labrum (black arrowhead in Fig. 2h). Although it may appear as if expression of Pt-foxQ2 could be found in the chelicerae (dotted outlines in Fig. $2 \mathrm{~h}$ ), the expression was in fact located underneath the identified in this spider. Number on branches indicates posterior probabilities as calculated by MrBayes

chelicerae and corresponded to the domains located next to the stomodeum at earlier stages (white arrowheads in Fig. 2e, f). The same was true for the complex expression in the neurogenic ectoderm, which is likely only expressed in the neurogenic ectoderm, but not in the epidermal tissue above. The complex expression pattern showed that Pt-foxQ2 was expressed in the anterior tip of the early germband and later in the neurogenic and non-neurogenic ectoderm and the labrum.

\section{Downregulation of $P t$-foxQ2 results in labrum defects}

In most species, fox $Q 2$ is downstream of $\operatorname{six} 3$ in the aGRN. In contrast, mutual activation was found in $T$. castaneum indicating a gain of an upstream role in the aGRN (Kitzmann et al. 2017). Therefore, we sought to identify the level of conservation of this genetic interaction between these two transcription factors in the spider. There are two paralogs of six 3 in P. tepidariorum (Pt-six3.1 and Pt-six3.2) and their embryonic expression patterns had been described previously 

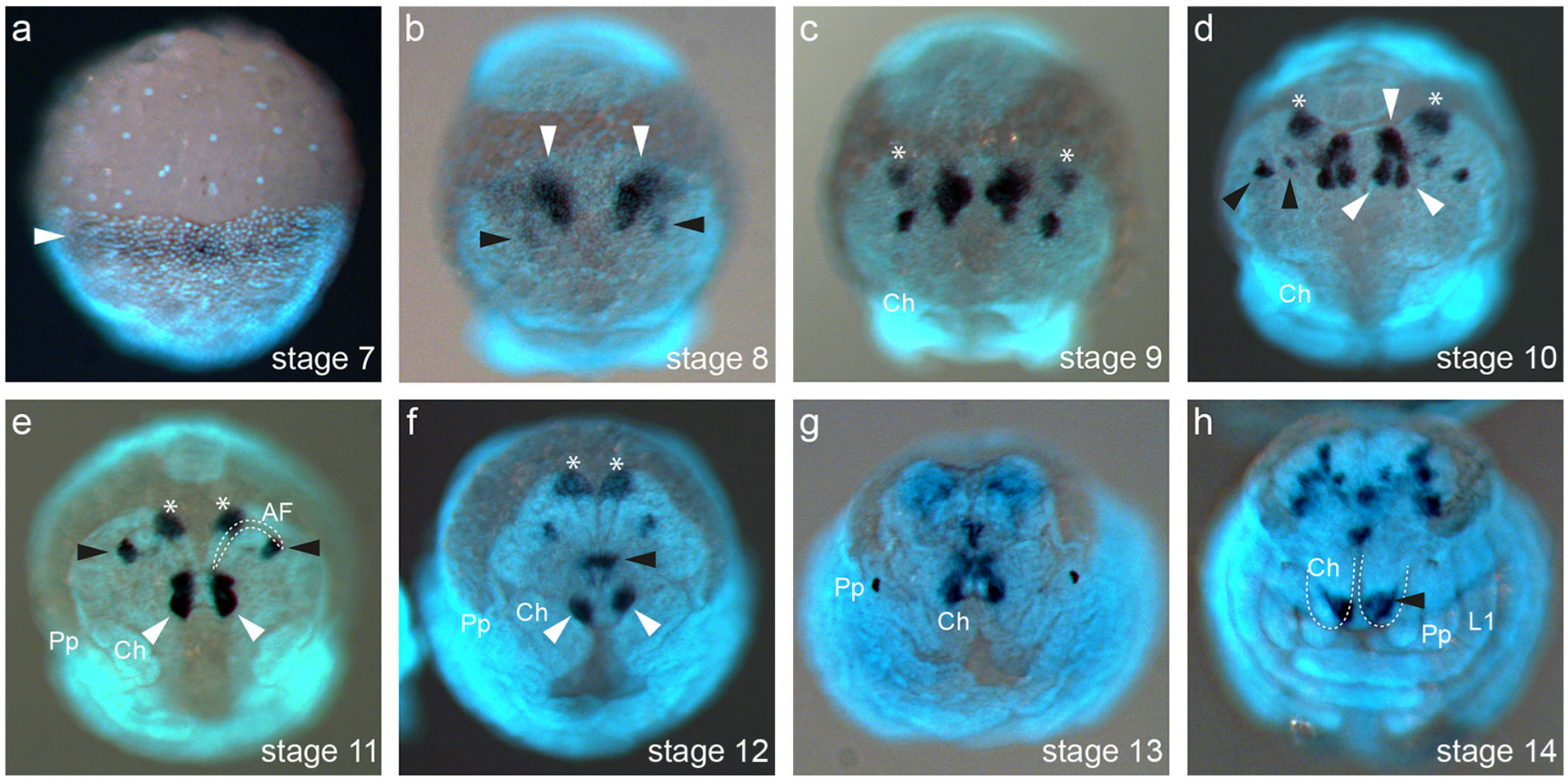

Fig. 2 Embryonic $P t$-fox $Q 2$ expression. After initial expression as a stripe at the anterior end of the embryo (white arrowhead in a), Pt-foxQ2 is found in several dynamic domains in the pre-cheliceral lobes $(\mathbf{b}-\mathbf{f})$. White and black arrowheads and asterisks mark the corresponding domains in all panels. From stage 11 onward, the head epidermis grows over the neurogenic ectoderm (anterior furrow (AF) in $\mathbf{e}$ ). At later stages, $P t$ -

(Schomburg et al. 2015). At late embryonic stages (stage 1012), Pt-six 3.1 is expressed in the neurogenic ectoderm and the labrum. Transcripts of Pt-six3.2 are detected in the labrum, stomodeum and in the anterior median region of the head. At stage 13, Pt-six3.2 is expressed in the primordia of the lateral eyes and in cells underneath the non-neurogenic ectoderm (Schomburg et al. 2015).

We performed parental RNAi (pRNAi) to downregulate the expression of Pt-foxQ2 and analyse the function of this transcription factor during embryogenesis. Likewise, we analysed the function of Pt-six 3.1 and Pt-six3.2 by RNAi to obtain information about a possible interaction between $P t$ foxQ2 and Pt-six3.1/six3.2. The analysis included data from females (cocoons 1-4 from 3 different females) that were either injected with dsRNAs (Pt-foxQ2, Pt-six3.1 or Ptsix3.2), or with injection buffer (negative control), and from one female spider that was not injected at all (wild type) (supplemental material Tab. S1). Each cocoon was checked for viable and dead embryos (referred to as Pt-gene $X^{\mathrm{RNAi}}$ embryos) and they were scored for morphological defects. We found that $P t$-foxQ $2^{\mathrm{RNAi}}, P t-\operatorname{six} 3.1^{\mathrm{RNAi}}$ and $P t$ six3. $2^{\mathrm{RNAi}}$ embryos showed no cuticle defects. They were able to develop until the embryo started to eclose. However, most of the embryos were unable to hatch from the egg membrane indicating an essential function of those genes. Instead, the membrane remained intact and the embryos stayed at this developmental stage for several days before drying out. Fifty-

foxQ2 transcripts are found in the labrum and the brain $(\mathbf{g}, \mathbf{h})$. Expression is not in the chelicerae but below of them (black arrowhead in $\mathbf{h}$ ). All embryos shown in frontal view. Developmental stages are indicated in the lower right-hand corner. AF: anterior furrow; Pp: pedipalps; $\mathrm{Ch}$ : chelicerae; L1: first walking leg)

nine percent of the Pt-foxQ2 ${ }^{\mathrm{RNAi}}$ embryos did not hatch, and the equivalent phenotype was observed for $43 \%$ in $P t$ six $3.1^{\mathrm{RNAi}}$, and for $24 \%$ in Pt-six $3.2^{\mathrm{RNAi}}$ embryos in the first cocoon. This phenotype increased in subsequent cocoons (99-100\% for Pt-foxQ2 $2^{\mathrm{RNAi}}$ embryos, $70-84 \%$ for $P t$ six3.1 ${ }^{\mathrm{RNAi}}$ embryos, $66-73 \%$ for Pt-six $3.2^{\mathrm{RNAi}}$ embryos) (Fig. 3a). Three to $36 \%$ of the negative control embryos did not hatch or were dead (Fig. 3a) which was similar to the rate observed in previous studies (Turetzek et al. 2015). While we did not observe cuticle phenotypes in any of our treatments, we did find morphological differences in the Pt-fox $Q 2^{R N A i}$ embryos at stages 10 (77\% in cocoon 1 and $88 \%$ in cocoon $2-4)$ and 11 (40\% in cocoon 1 and 50\% in cocoon 2-4). In $38 \%$ (cocoon 1) and in $45 \%$ (cocoon 2-4) of the injected embryos, the labrum was strongly reduced or completely missing (Fig. 3b; Fig. 4b-h).

\section{Interaction between Pt-foxQ2, Pt-six3.1 and Pt-six3.2}

In order to test whether Pt-foxQ2, Pt-six3.1 and Pt-six3.2, respectively, interact with one another, we performed $P t$ foxQ2 in situ hybridisations in Pt-six3.1 $1^{\mathrm{RNAi}}$ and $P t$ six3. $2^{\mathrm{RNAi}}$ embryos and vice versa. We found RNAi against $P t$-fox $Q 2$ had no effect on the expression of either of the Ptsix 3 paralogs, with the exception of the labrum, which normally expresses both Pt-six 3.1 and Pt-six3.2, but was missing after Pt-foxQ2 RNAi (Fig. 4; note that the embryo in b has an 

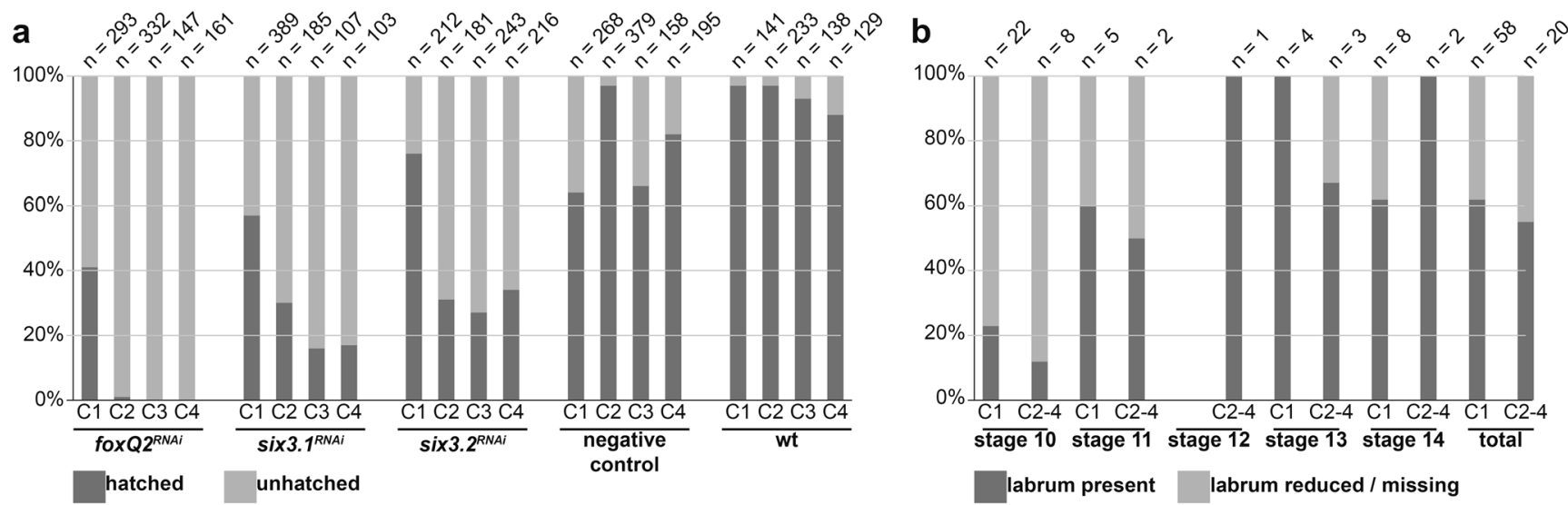

Fig. 3 Quantitative analysis of RNAi phenotypes. Overview over hatch rates in cocoons of dsRNA injected female spiders and controls (a). Overview of observed labrum defects in Pt-fox $Q 2^{R N A i}$ embryos by

overall weaker staining). The morphological defect served as internal control that the RNAi experiment had worked efficiently in these embryos. However, we cannot exclude that the level of downregulation may have been insufficient for an effect on Pt-six3.1 and Pt-six3.2 expression.

In the converse experiment, RNAi against neither Pt-six3.1 nor Pt-six3.2 showed any apparent morphological differences between wild-type and injected embryos (Fig. 5). The expression of Pt-foxQ2, however, was altered. At stage 10, the most anterior of the tripartite median domain (white arrowheads in Fig. 2d) was clearly missing after injection with either Pt-
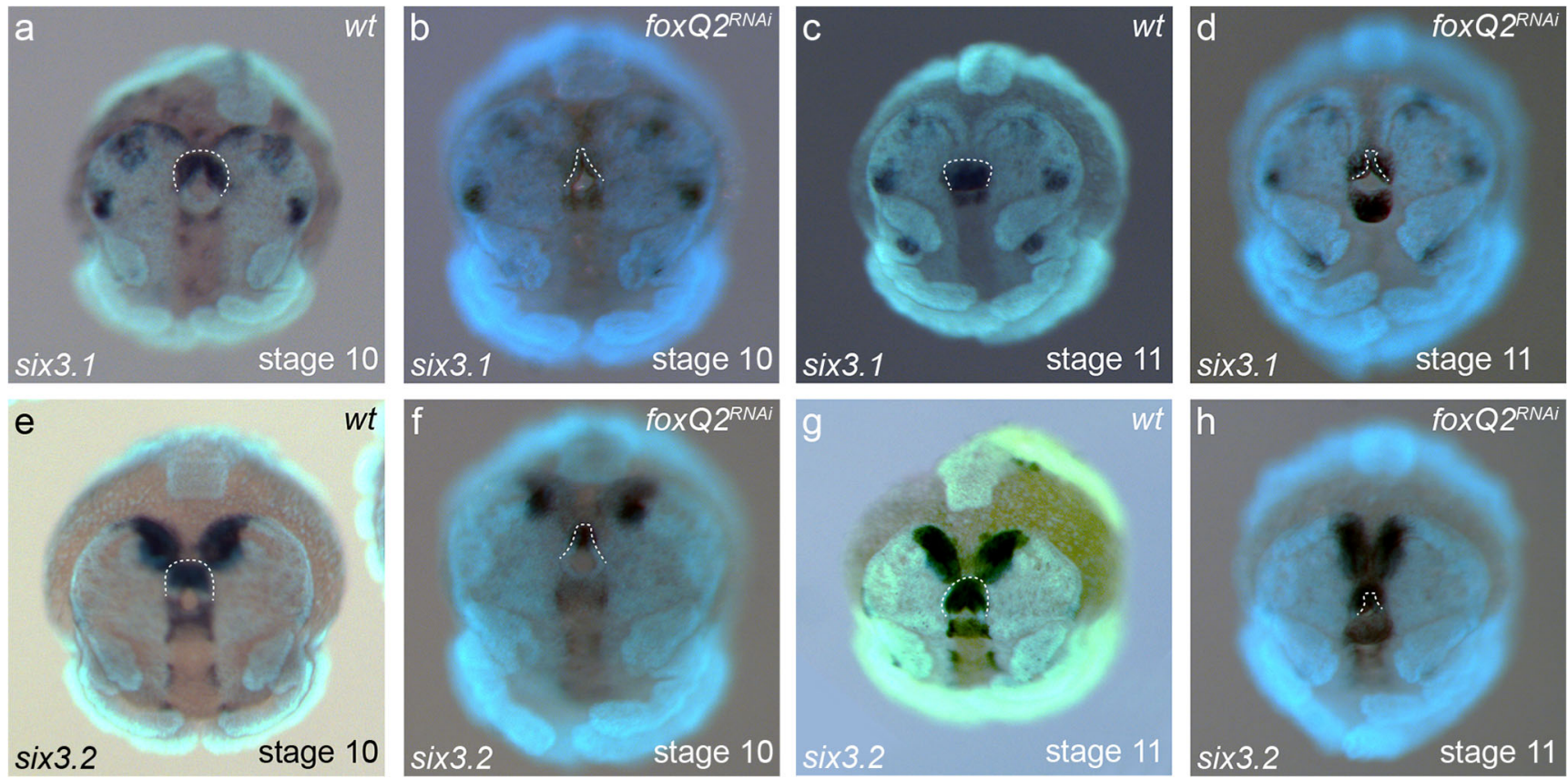

Fig. 4 Pt-six 3 expression in Pt-fox $Q 2^{R N A i}$ embryos. Expression of $P t$ six3.1 in Pt-foxQ2 $2^{R N A i}$ embryos (a-d). Expression of Pt-six3.2 in Ptfox $Q 2^{R N A i}$ embryos $(\mathbf{e}-\mathbf{h})$. There were no qualitative changes in expression of Pt-six 3 paralogs in Pt-fox $Q 2^{R N A i}$ embryos (note that the overall staining in embryo in $\mathrm{b}$ is weaker due to experimental conditions). After Pt-foxQ2 RNAi, the labrum (dotted lines in all

developmental stage and cocoons (b). Note that cocoons 2-4 showed similar strength (as measured by the hatch rate) and were therefore pooled for the in situ hybridization

six3.1 or Pt-six3.2 dsRNA (asterisks in Fig. 5a, c). At the later stages (Fig. 5b, d), it was difficult to make exact statements about the nature of the changes of expression in the RNAi phenotype, due to the complex dynamic of the expression patterns and the morphological rearrangements during those stages. However, it is clear that the Pt-foxQ2 expression domain around the stomodeum was reduced in the RNAi experiments, compared to the wild-type staining (white arrowheads in Fig. 2e). The domain was probably also lacking at stage 11, but this was less clear because at that stage, the two domains had fused in wild type (Fig. 2e).

panels) was reduced or missing leading to a secondary reduction of the Pt-foxQ2-positive tissue. All embryos shown in frontal view. Developmental stages are indicated in the lower right-hand corner, the stained gene in the lower left-hand corner and treatment in the upper righthand corner 

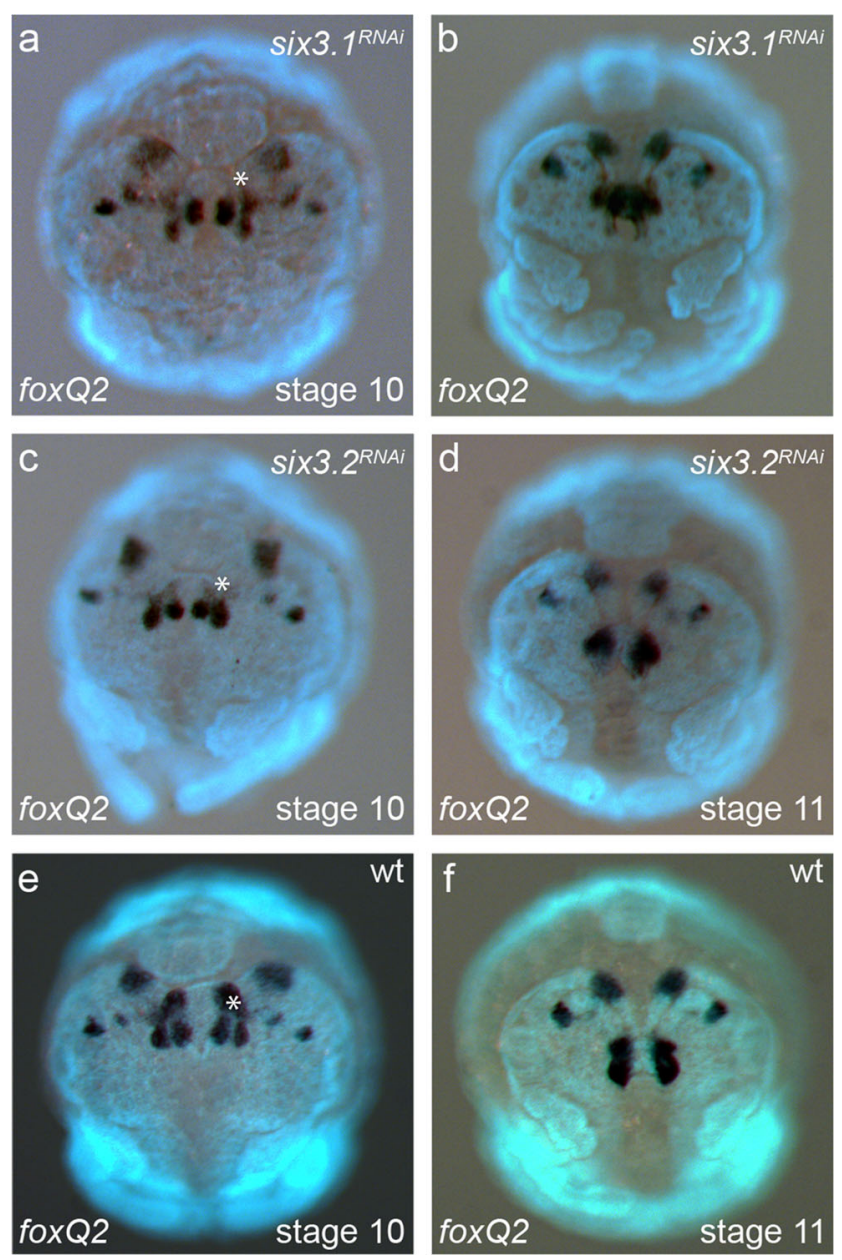

Fig. 5 Pt-foxQ2 expression in Pt-six3.1/3.2 $2^{R N A i}$ embryos. Expression of $P t$-foxQ2 in Pt-six3.1 $1^{R N A i}$ embryos (a, b). Expression of Pt-foxQ2 in Ptsix $3.2^{R N A i}$ embryos (c, d). In both treatments, embryos lacked the most anterior expression domain in the anterior median region (asterisks in $\mathbf{a}, \mathbf{c}$ ) at stage 10 . The domain around the stomodeum at stage 11 was reduced (b, d). Wild-type embryos of stage 10 and 11 are shown for comparison. All embryos shown in frontal view. Developmental stages are indicated in the lower right-hand corner, the stained gene in the lower left-hand corner and treatment in the upper right-hand corner

\section{Discussion}

\section{Pt-foxQ2 as a conserved anterior marker and important factor in labrum and neural development}

Here, we present the first expression and functional study of a foxQ2 homolog in chelicerates. Like in all metazoan animals analysed so far (Yu et al. 2003; Tu et al. 2006; Yaguchi et al. 2008; Yankura et al. 2010; Range and Wei 2016; Fritzenwanker et al. 2014; Marlow et al. 2014; Santagata et al. 2012; Lee and Frasch 2004; Kitzmann et al. 2017; Sinigaglia et al. 2013; Chevalier et al. 2006), it is expressed as a patterning gene in the aGRN in the early embryo. Later in development, Pt-foxQ2 is expressed in various regions of the neurogenic ectoderm, which suggests its involvement in neural development might be a conserved aspect among arthropods (Kitzmann et al. 2017; Hunnekuhl and Akam 2014). Furthermore, PtfoxQ2 has a similar effect on the development of the labrum as seen in T. castaneum (Kitzmann et al. 2017). This hints at a conserved role for fox $Q 2$ in the development of epidermal head structures in arthropods in contrast to other metazoan lineages where gene knockdown did not reveal strong epidermal defects (Yaguchi et al. 2008; Sinigaglia et al. 2013).

\section{The two Pt-six3 paralogs appear to compensate for each other functions in $P$. tepidariorum}

Loss of Pt-six3.1/3.2 function in P. tepidariorum showed no deleterious effect in the anterior median region in contrast to T. castaneum, where this whole region including the labrum is deleted (Posnien et al. 2011). This was unexpected because in all animals studied so far, six 3 acted upstream to or on the same level as foxQ2. However, P. tepidariorum has two six 3 paralogs with overlapping expression in the anterior median region (compare Fig. 4a and e), which could lead to a compensatory effect. One hint for compensatory function is the elevated portion of hatched animals in both Pt-six3.1/3.2 treatments (about $30 \%$ in the second clutch) compared to the PtfoxQ2 treatment (1\% in the second clutch). Unfortunately, double RNAi has so far not been successful in P. tepidariorum.

\section{An evolutionary playground of anterior interactions}

foxQ2 and six3 are both prominent factors in anterior development and are commonly co-expressed during embryogenesis and show quite different interactions in different animal species (Fritzenwanker et al. 2014; Hunnekuhl and Akam 2014; Marlow et al. 2014; Martín-Durán and Hejnol 2015; Santagata et al. 2012; Sinigaglia et al. 2013; Tu et al. 2006; Wei et al. 2009; Range and Wei 2016; Kitzmann et al. 2017). In all studied animals but in insects, six 3 is clearly upstream of foxQ2 in the aGRN. In T. castaneum, in contrast, Tc-foxQ2 gained a more upstream role activating $T c$-six 3 . Hence, both genes activate each other and knockdown of either gene leads to similar epidermal phenotypes (i.e. loss of the labrum) (Kitzmann et al. 2017). It had remained unclear whether this interaction was conserved in arthropods or whether it evolved later in the lineages leading to the insects.

As expected, regulation of Pt-foxQ2 by Pt-six3.1/3.2 was observed in P. tepidariorum. However, the RNAi experiments did not provide any evidence for $P t$-foxQ2 being required for $P t$-six3.1/3.2 expression as the expression of both Pt-six 3 paralogs remained unchanged in Pt-fox $Q 2^{R N A i}$ embryos, except for a secondary reduction due to the 


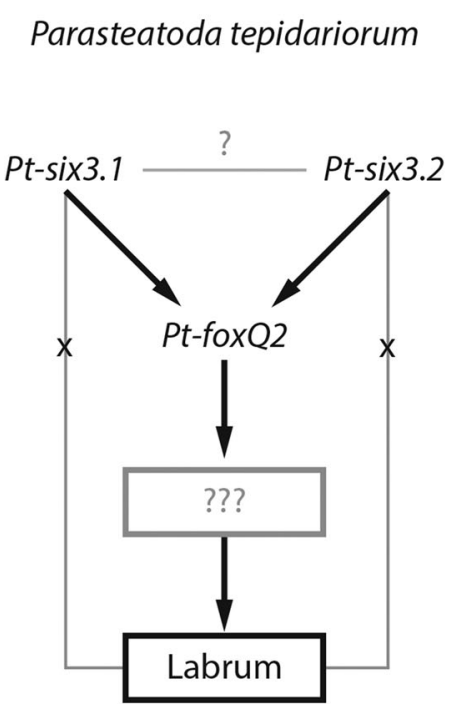

Fig. 6 Schematic overview of Pt-foxQ2 interaction with the Pt-six 3 paralogs. a Both Pt-six 3 paralogs are required for proper Pt-foxQ2 expression while $P t$-foxQ2 RNAi did not lead to alterations in neither of the $P t$-six 3 paralogs. These results place $P t$-foxQ2 downstream of Pt-six 3 . Only the knockdown of $P t-f o x Q 2$ led to morphologically visible reduction or loss of the labrum, while the single RNAis targeting either of the Pt-six 3 paralogs alone did result in no morphological alterations.

missing tissue of the labrum. We cannot exclude that we do not see an effect on Pt-six3.1/six3.2 expression because the RNAi embryos might still have residual transcripts sufficient for this activation. However, the strong RNAi effect on the labrum argues against this scenario. Hence, assuming that a $P t$-foxQ2 does not activate nor repress $P t$-six3.11 3.2 in P. tepidariorum, we suggest that the spider situation reflects the ancestral condition (i.e. six 3 upstream of foxQ2) while in the lineage leading to the insects fox $Q 2$ gained a more upstream role by activating six 3 expression.

\section{Conclusion}

Our expression analysis adds Pt-foxQ2 to the list of genes active in the early anterior neurogenic ectoderm in spiders. Our knockdown experiments identify both $P t$-foxQ2 and Pt-six3.1/3.2 as genes required for labrum development in P. tepidariorum (Fig. 6). This indicates that the epidermal patterning function of foxQ2 is an ancestral feature of arthropods. Further, we propose that Pt-six3.1/3.2 is upstream with respect to Pt-foxQ2 (Fig. 6), although we cannot rule out that Pt-foxQ2 RNAi knockdown was not strong enough to see an effect on the other genes. We propose that the interactions of the spider aGRN are conserved with those of other animals and suggest that fox $Q 2$ orthologs gained a more upstream role rather recently in the lineage leading to the insects.

\section{Tribolium castaneum}

I
I
I
I
I
I
I
I
I
I
I
I
I
I
I
I
I
I
I
I
I

Further data on labrum development are missing in spiders. b The beetle foxQ2 ortholog, in contrast, is required for proper expression of Tc-six 3 forming a mutual activation loop. Based on data from other species this indicates that fox $Q 2$ gained a more upstream role in the lineage leading to insects. Interactions from T. castaneum are taken from Siemanowski et al. 2015, Kittelmann et al. 2013 and Kitzmann et al. 2017

Acknowledgements We thank Natascha Turetzek, Peter Kitzmann and Nico Posnien for their assistance during the experimental process and helpful discussions.

Funding Information Open Access funding provided by Projekt DEAL.

Open Access This article is licensed under a Creative Commons Attribution 4.0 International License, which permits use, sharing, adaptation, distribution and reproduction in any medium or format, as long as you give appropriate credit to the original author(s) and the source, provide a link to the Creative Commons licence, and indicate if changes were made. The images or other third party material in this article are included in the article's Creative Commons licence, unless indicated otherwise in a credit line to the material. If material is not included in the article's Creative Commons licence and your intended use is not permitted by statutory regulation or exceeds the permitted use, you will need to obtain permission directly from the copyright holder. To view a copy of this licence, visit http://creativecommons.org/licenses/by/4.0/.

\section{References}

Akiyama-Oda Y, Oda H (2006) Axis specification in the spider embryo: dpp is required for radial-to-axial symmetry transformation and sog for ventral patterning. Development 133:2347-2357. https://doi.org/ 10.1242/dev.02400

Benayoun BA, Caburet S, Veitia RA (2011) Forkhead transcription factors: key players in health and disease. Trends Genet 27:224-232. https://doi.org/10.1016/j.tig.2011.03.003

Carlsson P, Mahlapuu M (2002) Forkhead transcription factors: key players in development and metabolism. Dev Biol 250:1-23. https://doi.org/10.1006/dbio.2002.0780

Chevalier S, Martin A, Leclère L, Amiel A, Houliston E (2006) Polarised expression of FoxB and FoxQ2 genes during development of the 
hydrozoan Clytia hemisphaerica. Dev Genes Evol 216:709-720. https://doi.org/10.1007/s00427-006-0103-6

Fritzenwanker JH, Gerhart J, Freeman RM, Lowe CJ (2014) The Fox/ Forkhead transcription factor family of the hemichordate Saccoglossus kowalevskii. EvoDevo 5:1-26. https://doi.org/10. 1186/2041-9139-5-17

He B, Buescher M, Farnworth MS et al (2019) An ancestral apical brain region contributes to the central complex under the control of foxQ2 in the beetle Tribolium. eLife 8. https://doi.org/10.7554/eLife.49065

Hilbrant M, Damen WGM, McGregor AP (2012) Evolutionary crossroads in developmental biology: the spider Parasteatoda tepidariorum. Development 139:2655-2662. https://doi.org/10. 1242/dev.078204

Hunnekuhl VS, Akam M (2014) An anterior medial cell population with an apical-organ-like transcriptional profile that pioneers the central nervous system in the centipede Strigamia maritima. Dev Biol 396: 136-149. https://doi.org/10.1016/j.ydbio.2014.09.020

Kaestner KH, Knöchel W, Martínez DE (2000) Unified nomenclature for the winged helix/forkhead transcription factors. Genes Dev 14:142 146. https://doi.org/10.1101/gad.14.2.142

Kittelmann S, Ulrich J, Posnien N, Bucher G (2013) Changes in anterior head patterning underlie the evolution of long germ embryogenesis. Dev Biol 374(1):174-184

Kitzmann P, Weißkopf M, Schacht MI, Bucher G (2017) A key role for foxQ2 in anterior head and central brain patterning in insects. Development 144:2969-2981. https://doi.org/10.1242/dev.147637

Lee H-H, Frasch M (2004) Survey of forkhead domain encoding genes in the Drosophila genome: classification and embryonic expression patterns. Dev Dyn 229:357-366. https://doi.org/10.1002/dvdy. 10443

Leite DJ, Baudouin-Gonzalez L, Iwasaki-Yokozawa S, LozanoFernandez J, Turetzek N, Akiyama-Oda Y, Prpic NM, Pisani D, Oda H, Sharma PP, McGregor A (2018) Homeobox gene duplication and divergence in arachnids. Mol Biol Evol 35:2240-2253

Lowe CJ, Wu M, Salic A et al (2003) Anteroposterior patterning in hemichordates and the origins of the chordate nervous system. Cell 113:853-865. https://doi.org/10.1016/S0092-8674(03)00469-0

Marlow H, Tosches MA, Tomer R et al (2014) Larval body patterning and apical organs are conserved in animal evolution. BMC Biol 12:1. https://doi.org/10.1186/1741-7007-12-7

Martín-Durán JM, Hejnol A (2015) The study of Priapulus caudatus reveals conserved molecular patterning underlying different gut morphogenesis in the Ecdysozoa. BMC Biol 13:29. https://doi.org/ 10.1186/s12915-015-0139-z

Mittmann B, Wolff C (2012) Embryonic development and staging of the cobweb spider Parasteatoda tepidariorum C. L. Koch, 1841 (syn.: Achaearanea tepidariorum; Araneomorphae; Theridiidae). Dev Genes Evol 222:189-216. https://doi.org/10.1007/s00427-0120401-0

Paese CLB, Schoenauer A, Leite DJ et al (2018) A SoxB gene acts as an anterior gap gene and regulates posterior segment addition in a spider. eLife 7:e37567. https://doi.org/10.7554/eLife. 37567

Pechmann M, Benton MA, Kenny NJ et al (2017) A novel role for Ets4 in axis specification and cell migration in the spider Parasteatoda tepidariorum. eLife 6:e27590. https://doi.org/10. 7554/eLife. 27590

Pechmann M, Prpic N-M (2009) Appendage patterning in the south American bird spider Acanthoscurria geniculata (Araneae: Mygalomorphae). Dev Genes Evol 219:189-198. https://doi.org/ 10.1007/s00427-009-0279-7

Pechmann M, Schwager EE, Turetzek N, Prpic N-M (2015) Regressive evolution of the arthropod tritocerebral segment linked to functional divergence of the Hox gene labial. Proc R Soc B Biol Sci 282: 20151162. https://doi.org/10.1098/rspb.2015.1162
Posnien N, Koniszewski NDB, Hein HJ, Bucher G (2011) Candidate gene screen in the red flour beetle Tribolium reveals Six3 as ancient regulator of anterior median head and central complex development. PLoS Genet 7:e1002416. https://doi.org/10.1371/journal.pgen. 1002416

Posnien N, Zeng V, Schwager EE et al (2014) A comprehensive reference Transcriptome resource for the common house spider Parasteatoda tepidariorum. PLoS One 9. https://doi.org/10.1371/journal.pone. 0104885

Prpic N-M, Schoppmeier M, Damen WGM (2008) Whole-mount in situ hybridization of spider embryos. Cold Spring Harb Protoc 2008:pdb.prot5068-pdb.prot5068. https://doi.org/10.1101/pdb. prot5068

Range RC, Wei Z (2016) An anterior signaling center patterns and sizes the anterior neuroectoderm of the sea urchin embryo. Development. https://doi.org/10.1242/dev.128165

Ronquist F, Teslenko M, van der Mark P, Ayres DL, Darling A, Höhna S, Larget B, Liu L, Suchard MA, Huelsenbeck JP (2012) MrBayes 3.2: efficient Bayesian phylogenetic inference and model choice across a large model space. Syst Biol 61:539-542. https://doi.org/10.1093/ sysbio/sys029

Santagata S, Resh C, Hejnol A, Martindale MQ, Passamaneck YJ (2012) Development of the larval anterior neurogenic domains of Terebratalia transversa (Brachiopoda) provides insights into the diversification of larval apical organs and the spiralian nervous system. EvoDevo 3:3. https://doi.org/10.1186/2041-9139-3-3

Schomburg C, Turetzek N, Schacht MI, Schneider J, Kirfel P, Prpic NM, Posnien N (2015) Molecular characterization and embryonic origin of the eyes in the common house spider Parasteatoda tepidariorum. EvoDevo 6:1-14. https://doi.org/10.1186/s13227-015-0011-9

Schwager EE, Pechmann M, Feitosa NM, McGregor A, Damen WG (2009) Hunchback functions as a segmentation gene in the spider Achaearanea tepidariorum. Curr Biol 19:1333-1340. https://doi.org/ 10.1016/j.cub.2009.06.061

Schwager EE, Sharma PP, Clarke T, Leite DJ, Wierschin T, Pechmann M, Akiyama-Oda Y, Esposito L, Bechsgaard J, Bilde T, Buffry AD, Chao H, Dinh H, Doddapaneni H, Dugan S, Eibner C, Extavour CG, Funch P, Garb J, Gonzalez LB, Gonzalez VL, Griffiths-Jones S, Han Y, Hayashi C, Hilbrant M, Hughes DST, Janssen R, Lee SL, Maeso I, Murali SC, Muzny DM, Nunes da Fonseca R, Paese CLB, Qu J, Ronshaugen M, Schomburg C, Schönauer A, Stollewerk A, Torres-Oliva M, Turetzek N, Vanthournout B, Werren JH, Wolff C, Worley KC, Bucher G, Gibbs RA, Coddington J, Oda H, Stanke M, Ayoub NA, Prpic NM, Flot JF, Posnien N, Richards S, McGregor A (2017) The house spider genome reveals an ancient whole-genome duplication during arachnid evolution. BMC Biol 15:62. https://doi. org/10.1186/s12915-017-0399-x

Siemanowski J, Richter T, Dao VA, Bucher G (2015) Notch signaling induces cell proliferation in the labrum in a regulatory network different from the thoracic legs. Dev Biol 408:164-177

Sievers F, Higgins DG (2014) Clustal omega, accurate alignment of very large numbers of sequences. In: Russell DJ (ed) Multiple Sequence Alignment Methods. Humana Press, Totowa, pp 105-116

Sievers F, Wilm A, Dineen D, Gibson TJ, Karplus K, Li W, Lopez R, McWilliam H, Remmert M, Söding J, Thompson JD, Higgins DG (2011) Fast, scalable generation of high-quality protein multiple sequence alignments using Clustal omega. Mol Syst Biol 7:539. https://doi.org/10.1038/msb.2011.75

Sinigaglia C, Busengdal H, Leclère L et al (2013) The Bilaterian head patterning gene six $3 / 6$ controls aboral domain development in a cnidarian. PLoS Biol 11. https://doi.org/10.1371/journal.pbio. 1001488

Steinmetz PR, Urbach R, Posnien N et al (2010) Six3 demarcates the anterior-most developing brain region in bilaterian animals. EvoDevo 1:1. https://doi.org/10.1186/2041-9139-1-14 
Tosches MA, Arendt D (2013) The bilaterian forebrain: an evolutionary chimaera. Curr Opin Neurobiol 23:1080-1089. https://doi.org/10. 1016/j.conb.2013.09.005

Tu Q, Brown CT, Davidson EH, Oliveri P (2006) Sea urchin Forkhead gene family: phylogeny and embryonic expression. Dev Biol 300: 49-62. https://doi.org/10.1016/j.ydbio.2006.09.031

Turetzek N, Pechmann M, Schomburg C et al (2015) Neofunctionalization of a duplicate dachshund gene underlies the evolution of a novel leg segment in arachnids. Mol Biol Evol 33: $109-121$

Uhlenhaut NH, Treier M (2011) Forkhead transcription factors in ovarian function. Reprod 142:489-495. https://doi.org/10.1530/REP-110092

Wei Z, Yaguchi J, Yaguchi S, Angerer RC, Angerer LM (2009) The sea urchin animal pole domain is a Six3-dependent neurogenic patterning center. Development 136:1179-1189. https://doi.org/10.1242/ dev.032300
Yaguchi S, Yaguchi J, Angerer RC, Angerer LM (2008) A Wnt-FoxQ2nodal pathway links primary and secondary axis specification in sea urchin embryos. Dev Cell 14:97-107. https://doi.org/10.1016/j. devcel.2007.10.012

Yankura KA, Martik ML, Jennings CK, Hinman VF (2010) Uncoupling of complex regulatory patterning during evolution of larval development in echinoderms. BMC Biol 8:143. https://doi.org/10.1186/ 1741-7007-8-143

Yu J-K, Holland ND, Holland LZ (2003) AmphiFoxQ2, a novel winged helix/forkhead gene, exclusively marks the anterior end of the amphioxus embryo. Dev Genes Evol 213:102-105. https://doi.org/10. 1007/s00427-003-0302-3

Publisher's note Springer Nature remains neutral with regard to jurisdictional claims in published maps and institutional affiliations. 